\title{
Ring Opening of Epoxy Fatty Esters by Nucleophile to Form the Derivatives of Substituted $\beta$-Amino Alcohol
}

\author{
Himani Varshney, Aiman Ahmad, Abdul Rauf* \\ Department of Chemistry, Aligarh Muslim University, Aligarh, India. \\ Email: *abduloafchem@gmail.com \\ Received May $14^{\text {th }}, 2013$; revised June $14^{\text {th }}, 2013$; accepted June $21^{\text {st }}, 2013$ \\ Copyright (C) 2013 Himani Varshney et al. This is an open access article distributed under the Creative Commons Attribution License, \\ which permits unrestricted use, distribution, and reproduction in any medium, provided the original work is properly cited.
}

\begin{abstract}
The nucleophilic ring opening of epoxy fatty esters was carried out using the amino-1,2,4-triazole to yield substituted derivatives of $\beta$-amino alcohol. The synthesis of the substituted beta amino alcohols has been achieved by refluxing equimolar quantities of long chain epoxy esters (epoxy fatty esters) and 4-amino-1,2,4-triazole in dichloromethane to yield following compounds, methyl 10-(4'-amino-1',2',4'-triazole)-11-hydroxy undecanoate (V), methyl 9-(4'-amino-1', 2',4'-triazole)-10-hydroxy octadecanoate (VI), methyl 9-(4'-amino-1',2',4'-triazole)-10,12-dihydroxy octadecanoate (VII), methyl 12-(4'-amino-1',2',4'-triazole)-9,13-dihydroxy octadecanoate (VIII). Epoxides of esters of fatty acids were obtained by reaction of esters of fatty acids with m-chloroperbenzoic acid. All the newly synthesized compounds were characterized by IR, ${ }^{1} \mathrm{H} \mathrm{NMR},{ }^{13} \mathrm{C}$ NMR and mass spectrometry.
\end{abstract}

Keywords: Triazole; Epoxide of Ester of Fatty Acid; Ring Opening

\section{Introduction}

$\beta$-Amino alcohols are very important class of organic compound and this type of organic moiety is found in various biologically active alkaloids and peptides [1]. These $\beta$-amino alcohols are synthesized by ring opening reaction of epoxides with a variety of electrophilic and nucleophilic reagents under microwave assisted reaction condition as well as at the elevated temperature [2] and long reaction time. The ring opening of epoxide has been also carried out under mild conditions, but this needs expensive catalysts, large excess of reagents and solvents [3].

In the field of organic synthesis, the epoxides are important intermediates, because of the high reactivity of their three member oxirane ring [4]. The inherent polarity and the maximum strain in the epoxide ring, allows the ring to react with a variety of Lewis acids nucleophilic reagents, such as amines, alcohols and thiols, to form corresponding $\beta$-amino alcohols, 1,2-diols [5], and $\beta$ hydroxysulfides [6], respectively, catalyzed by various Lewis acids. $\beta$-Amino alcohols are medicinally very important as they possess antimalarial parasitic activities [7] and also used in the synthesis of antihypertensive drugs [8].

${ }^{*}$ Corresponding author.
$\beta$-Amino alcohols are easily converted into many other molecules such as amino acids and amino sugars [9]. A literature survey revealed that although various epoxy ring containing fatty acid esters have been used for the synthesis of a variety of oleochemicals [10] yet. Except the recent report by the Biswas et al. [11], there is no report on the ring opening of epoxides of fatty acid esters with amino triazole. Recently, ring opening of terminal fatty acid methyl esters with other nucleophilic reagents was reported in the literature [12]. Keeping in view, the above mentioned synthetic and biological properties of $\beta$-amino alcohols, we have planned to synthesize these derivatives of fatty acids. Therefore, we report here the synthesis of substituted $\beta$-amino alcohols, synthesized by ring opening reaction of fatty acid epoxides with 4-amino-1,2,4-triazole in dichloromethane.

\section{Result and Discussion}

Various heterocyclic compounds from fatty esters bearing terminal or internal double bond were reported as potent dipolarophiles. All these compounds were obtained in good yield. In this paper, we report the ring opening reaction of epoxy fatty ester with a nucleophile, here, the amino group of the 4-amino-1,2,4-triazole act as nucleophile, to form the substituted $\beta$-amino alcohol 
presented in the Scheme 1. The ring opening reaction of terminal, $I$, and internal, II-IV, epoxy fatty esters with amino triazole resulted in the formation of inseparable mixtures, $V$-VIII, of $\beta$-amino alcohol in appreciable yield ( $68 \%$ to $74 \%)$. Generally, in the case of ring opening of epoxides in basic medium, the nucleophilic attack occurs from the less hindered side [13], to give a major product. Similar situation was observed in the case of terminal epoxy ester, $I$, there was a choice for the nucleophile to attack from the less hindered side therefore, product $V$ was formed as a major product. Another isomer may also be formed in trace amount, but could not be isolated. In the case of internal epoxy fatty esters, II-IV, both sides were equally hindered; therefore there was formation of inseparable mixtures, VI-VIII, which could not be isolated from one another, description of the compounds $V$-VIII were tabulated in Table 1. There was some possibility of intramolecular H-bonding (between N-H-O and $\mathrm{H}-\mathrm{O}-\mathrm{H}$ ); this situation was possible in both of the isomers that will have no effect on their separation. Because of the H-bonding, the peak of NH in IR spectrum became slightly broadened. Newly synthesized products, V-VIII, were characterized by IR, ${ }^{1} \mathrm{H}$ NMR, ${ }^{13} \mathrm{C}$ NMR and mass spectroscopy. A detailed description of the compound $V$ is discussed. IR spectrum of this compound showed the characteristic peaks between $3438-3247 \mathrm{~cm}^{-1}$ and at $1736 \mathrm{~cm}^{-1}$. The ${ }^{1} \mathrm{H}$ NMR spectra of the compound showed some characteristic peaks. A triplet of doublet at $\delta 7.86$ for $\mathrm{NH}$ proton, two multiplets at $\delta 7.48$ and 7.33 for five membered ring protons, a broad singlet at $\delta 3.82$ for the hydroxyl group, and one sharp singlet at $\delta 3.61$ for the ester group. The ${ }^{13} \mathrm{C}$ NMR spectrum showed the peak at $\delta 174.5$ for the carbonyl carbon and at $\delta 146.8$, 146.5 for triazole ring. Mass spectral results were supported the proposed structure.

\section{Experimental}

Undec-9-enoic acid and (9Z)-octadec-9-enoic acid were purchased commercially from Fluka Chemicals (Switzerland). (9Z, 12R)-12-hydroxy octadec-9-enoic acid (Ricinolic acid) and (12Z, 9R)-9-hydroxy octadec-12enoic acid (Isoricinolic acid) were extracted from naturally occurring seeds by Gunstone's partitioning method $[14,15]$.

\subsection{General Procedure for the Synthesis of Epoxy Fatty Ester (I-IV)}

The cis fatty ester epoxides were prepared according to the method of $[15,16]$.

\subsection{General Procedure for the Synthesis of Compound (V-VIII)}

$\beta$-Amino alcohols were synthesized from equimolar amounts of cis epoxy fatty ester $(0.1 \mathrm{mmol})$ and $4-$ amino-1,2,4-triazole $(0.1 \mathrm{mmol})$ in dichloromethane solvent $(30 \mathrm{~mL})$ and reflux it on water bath for $4-6 \mathrm{hr}$. Progress of the reaction was monitored by TLC and after

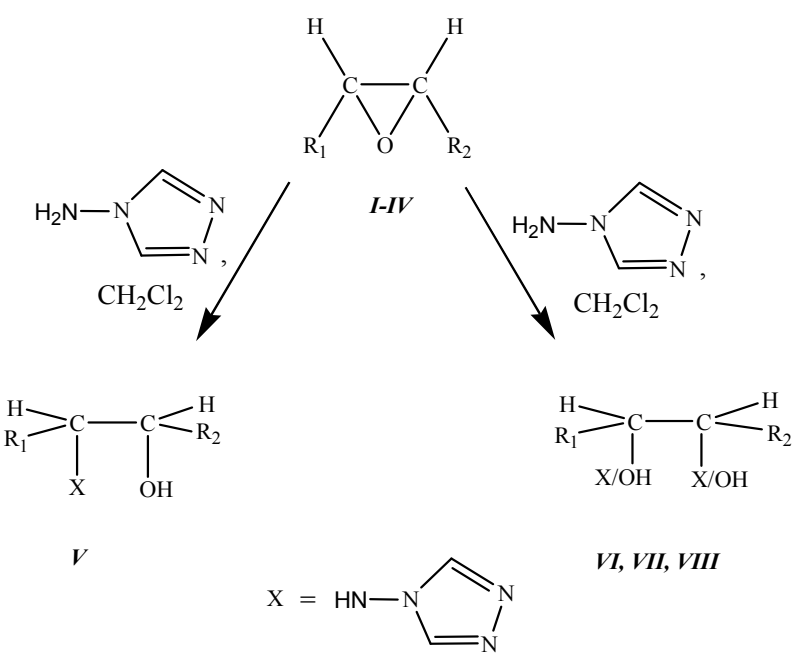

Scheme 1. Synthetic pathway for the synthesis of substituted $\beta$-amino alcohols $V$-VIII.

Table 1. Description of newly synthesized Compounds V-VIII.

\begin{tabular}{|c|c|c|c|c|}
\hline Comp. & $\mathrm{R}_{1}$ & $\mathrm{R}_{2}$ & Molecular formula & $\%$ yield \\
\hline$I, V$ & $\mathrm{H}$ & $\left(\mathrm{CH}_{2}\right)_{8}-\underset{\mathrm{O}}{\mathrm{C}}-\mathrm{OCH}_{3}$ & $\mathrm{C}_{14} \mathrm{H}_{26} \mathrm{O}_{3} \mathrm{~N}_{4}$ & 68 \\
\hline$I I, V I$ & $\mathrm{CH}_{3}-\left(\mathrm{CH}_{2}\right)_{7}$ & $\left(\mathrm{CH}_{2}\right)_{7}-\underset{\mathrm{O}}{\mathrm{C}}-\mathrm{OCH}_{3}$ & $\mathrm{C}_{21} \mathrm{H}_{40} \mathrm{O}_{3} \mathrm{~N}_{4}$ & 72 \\
\hline$I I I, V I I$ & $\mathrm{CH}_{3}-\left(\mathrm{CH}_{2}\right)_{5}-\underset{\mathrm{OH}}{\mathrm{CH}}-\mathrm{CH}_{2}$ & 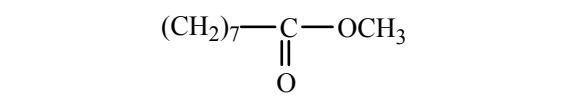 & $\mathrm{C}_{21} \mathrm{H}_{40} \mathrm{O}_{4} \mathrm{~N}_{4}$ & 74 \\
\hline$I V, V I I I$ & $\mathrm{CH}_{3}-\left(\mathrm{CH}_{2}\right)_{4}$ & $\mathrm{CH}_{2}-\mathrm{CH}_{2}-\underset{\mathrm{OH}}{\mathrm{CH}}-\left(\mathrm{CH}_{2}\right)_{7}-\underset{\|}{\mathrm{C}}-\mathrm{OCH}_{3}$ & $\mathrm{C}_{21} \mathrm{H}_{40} \mathrm{O}_{4} \mathrm{~N}_{4}$ & 70 \\
\hline
\end{tabular}


completion, the reaction mixture was cooled and worked up with diethyl ether-water. The ethereal solution was dried over anhydrous sodium sulfate $\left(\mathrm{Na}_{2} \mathrm{SO}_{4}\right)$ and concentrated in vacuo. An oily crude product was obtained in $68-74 \%$ yield, which was further purified by column chromatography using petrol-diethyl ether as eluent. Spectral data of the synthesized compounds are given below.

Methyl 11-(4'-amino-1',2',4'-triazole)-10-hydroxy undecanoate $(V)$ : IR (KBr): 3438 - $3247(\mathrm{OH}, \mathrm{NH}), 2928$ (C-H asym.), 2855 (C-H sym.), 1736 (ester). ${ }^{1} \mathrm{H}$ NMR $\left(400 \mathrm{MH}_{\mathrm{Z}}, \mathrm{CDCl}_{3}, \delta, \mathrm{ppm}\right): 7.86\left(\mathrm{td}, J_{\mathrm{NH}-\mathrm{CH}}=7.80,1.32\right.$ $\left.\mathrm{H}_{\mathrm{Z}}, 1 \mathrm{H}, \mathrm{N} H\right), 7.48(\mathrm{~m}, 1 \mathrm{H}$, ring $\mathrm{CH}), 7.33(\mathrm{~m}, 1 \mathrm{H}$, ring $\mathrm{CH}$ ), 3.82 (br. s, $1 \mathrm{H}, \mathrm{CHOH}), 3.61$ (s, 3H, OCH $\left.\mathrm{O}_{3}\right), 3.31$ $3.25(\mathrm{~m}, 1 \mathrm{H}, \mathrm{CHOH}), 3.24-3.20\left(\mathrm{~m}, 1 \mathrm{H}, H_{z} \mathrm{CH}-\mathrm{NH}\right)$, $3.20-3.15\left(\mathrm{~m}, 1 \mathrm{H}, H_{E} \mathrm{CHNH}\right), 2.23\left(\mathrm{t}, J=7.98 \mathrm{H}_{\mathrm{Z}}, 2 \mathrm{H}\right.$, $\left.\mathrm{CH}_{2} \mathrm{CO}\right), 1.53\left(\mathrm{~m}, 2 \mathrm{H}, \mathrm{CH}_{2} \mathrm{CH}_{2} \mathrm{CO}\right), 1.33(\mathrm{~m}, 2 \mathrm{H}$, $\mathrm{CH}_{2} \mathrm{CHCH}_{2}$ ), 1.18 (br. s, $\left.10 \mathrm{H}, \mathrm{CH}_{2}\left(\mathrm{CH}_{2}\right)_{5}\right),{ }^{13} \mathrm{C} \mathrm{NMR}$ $\left(100 \mathrm{MH}_{\mathrm{Z}}, \mathrm{CDCl}_{3}, \delta, \mathrm{ppm}\right): 174.5(\mathrm{C}=\mathrm{O}), 146.8,146.5$ (ring), 74.0, $71.5(\mathrm{CH}-\mathrm{OH}), 62.1(\mathrm{CH}-\mathrm{NH}), 51.0\left(\mathrm{OCH}_{3}\right)$, 38.9 - 20.7 (alkyl chain). MS (ESI): $\mathrm{m} / \mathrm{z}=320.1[\mathrm{M}+$ $\mathrm{Na}]^{+}$, Calculated $=321.3$.

Methyl 9-(4'-amino-1',2',4'-triazole)-10-hydroxy octadecanoate (VI): IR (KBr): 3479 - $3233(\mathrm{OH}, \mathrm{NH}), 2926$ (C-H asym.), 2858 (C-H sym.), 1737 (ester) ${ }^{1} \mathrm{H}$ NMR (400 $\left.\mathrm{MH}_{\mathrm{Z}}, \mathrm{CDCl}_{3}, \delta, \mathrm{ppm}\right): 7.92\left(\mathrm{td}, J_{\mathrm{NH}-\mathrm{CH}}=7.76,1.20\right.$ $\left.\mathrm{H}_{\mathrm{Z}}, 1 \mathrm{H}, \mathrm{NH}\right), 7.53(\mathrm{~m}, 1 \mathrm{H}$, ring $\mathrm{CH}), 7.38(\mathrm{~m}, 1 \mathrm{H}$, ring $\mathrm{CH}$ ), 3.88 (br. s, $1 \mathrm{H}, \mathrm{CHOH}), 3.62$ (s, 3H, OCH $\left.\mathrm{O}_{3}\right), 3.36$ 3.25 (m, 1H, methine $\mathrm{CH}$ ), 3.16-2.99 (m, 1H, methine $\mathrm{CH}), 2.28\left(\mathrm{t}, J=7.46 \mathrm{H}_{\mathrm{Z}}, 2 \mathrm{H}, \mathrm{CH}_{2} \mathrm{CO}\right), 1.56(\mathrm{~m}, 4 \mathrm{H}$, $\left.\mathrm{CH}_{2} \mathrm{CH}-\mathrm{CHCH}_{2}\right), 1.52\left(\mathrm{~m}, 2 \mathrm{H}, \mathrm{CH}_{2} \mathrm{CH}_{2} \mathrm{CO}\right), 1.41$ (m, $2 \mathrm{H}, \mathrm{CH}_{2} \mathrm{CHOH}$ ), 1.25 (br. s, $\left.18 \mathrm{H},\left(\mathrm{CH}_{2}\right)_{9}\right), 0.87$ ( t, $J=$ $\left.6.48 \mathrm{H}_{\mathrm{Z}}, 3 \mathrm{H}, \mathrm{CH}_{2} \mathrm{CH}_{3}\right) .{ }^{13} \mathrm{C} \mathrm{NMR}\left(100 \mathrm{MH}_{\mathrm{Z}}, \mathrm{CDCl}_{3}, \delta\right.$, ppm): $173.8(\mathrm{C}=\mathrm{O}), 148.7,145.6$ (ring), $74.4(\mathrm{CH}-\mathrm{OH})$, $71.5(\mathrm{CH}-\mathrm{NH}), 50.3\left(\mathrm{OCH}_{3}\right), 34.0$ - 22.7 (alkyl chain), $14.2\left(\mathrm{CH}_{3}\right)$. MS (ESI): $\mathrm{m} / \mathrm{z}=419.2[\mathrm{M}+\mathrm{Na}]^{+}$, Calculated $=419.4$.

Methyl 9-(4'-amino-1',2',4'-triazole)-10,12-dihydroxy octadecanoate (VII): IR (KBr): 3444 - $3233(\mathrm{OH}, \mathrm{NH})$, 2928 (C-H asym.), 2857 (C-H sym.), 1730 (ester). ${ }^{1} \mathrm{H}$ NMR (400 $\left.\mathrm{MH}_{\mathrm{Z}}, \mathrm{CDCl}_{3}, \delta, \mathrm{ppm}\right): 7.98\left(\mathrm{td}, J_{\mathrm{NH}-\mathrm{CH}}=7.80\right.$, $\left.1.24 \mathrm{H}_{\mathrm{Z}}, 1 \mathrm{H}, \mathrm{NH}\right), 7.54(\mathrm{~m}, 1 \mathrm{H}$, ring $\mathrm{CH}), 7.40(\mathrm{~m}, 1 \mathrm{H}$, ring $\mathrm{CH}$ ), 3.93-3.90 (br. s, $2 \mathrm{H}$, merged two $\mathrm{OH}$ Peaks), $3.71\left(\mathrm{~s}, 3 \mathrm{H}, \mathrm{OCH}_{3}\right), 3.68-3.51(\mathrm{~m}, 1 \mathrm{H}$, methine $\mathrm{CH}), 3.50$ - $3.35(\mathrm{~m}, 1 \mathrm{H}$, methine $\mathrm{CH}), 2.30\left(\mathrm{t}, J=8.20 \mathrm{H}_{\mathrm{Z}}, 2 \mathrm{H}\right.$, $\left.\mathrm{CH}_{2} \mathrm{CO}\right), 1.64\left(\mathrm{~m}, 4 \mathrm{H}, \mathrm{CH}_{2} \mathrm{CH}-\mathrm{CHCH}_{2}\right), 1.54(\mathrm{~m}, 2 \mathrm{H}$, $\left.\mathrm{CH}_{2} \mathrm{CH}_{2} \mathrm{CO}\right) 1.52$ (m, 2H, $\mathrm{CH}_{2} \mathrm{CHOH}$ ), 1.31 (br. s, $16 \mathrm{H}$, $\left.\left(\mathrm{CH}_{2}\right)_{8}\right), 0.87\left(\mathrm{t}, \mathrm{J}=6.20 \mathrm{H}_{\mathrm{Z}}, 3 \mathrm{H}, \mathrm{CH}_{2} \mathrm{CH}_{3}\right) .{ }^{13} \mathrm{C} \mathrm{NMR}$ $\left(100 \mathrm{MH}_{\mathrm{Z}}, \mathrm{CDCl}_{3}, \delta, \mathrm{ppm}\right) 172.9(\mathrm{C}=\mathrm{O}), 148.6,147.7$ (ring), 69.7, $69.0(\mathrm{CH}-\mathrm{OH}), 64.3(\mathrm{CH}-\mathrm{NH}), 50.8\left(\mathrm{OCH}_{3}\right)$, 38.9 - 20.7 (alkyl chain), $13.9\left(\mathrm{CH}_{3}\right)$. MS (ESI): $\mathrm{m} / \mathrm{z}=$ $435.2[\mathrm{M}+\mathrm{Na}]^{+}$, Calculated $=435.4$.

Methyl 12-(4'-amino-1',2',4'-triazole)-9,13-dihydroxy octadecanoate (VIII): IR (KBr): 3453 - 3227 (OH, NH),
2922 (C-H asym.), 2854 (C-H sym.), 1732 (ester). ${ }^{1} \mathrm{H}$ NMR (400 $\left.\mathrm{MH}_{\mathrm{Z}}, \mathrm{CDCl}_{3}, \delta, \mathrm{ppm}\right): 7.95\left(\mathrm{td}, J_{\mathrm{NH}-\mathrm{CH}}=7.76\right.$, $\left.1.21 \mathrm{H}_{\mathrm{Z}}, 1 \mathrm{H}, \mathrm{NH}\right), 7.55(\mathrm{~m}, 1 \mathrm{H}$, ring $\mathrm{CH}), 7.39(\mathrm{~m}, 1 \mathrm{H}$, ring $\mathrm{CH}$ ), 4.14-3.86 (br. s, $2 \mathrm{H}$, merged two $\mathrm{OH}$ peak), $3.72\left(\mathrm{~s}, 3 \mathrm{H}, \mathrm{OCH}_{3}\right), 3.68-3.45(\mathrm{~m}, 1 \mathrm{H}$, methine $\mathrm{CHOH})$, 3.45 - $3.36(\mathrm{~m}, 1 \mathrm{H}$, methine $\mathrm{CH}), 2.29\left(\mathrm{t}, J=8.94 \mathrm{H}_{\mathrm{Z}}, 2 \mathrm{H}\right.$, $\left.\mathrm{CH}_{2} \mathrm{CO}\right), 1.63\left(\mathrm{~m}, 4 \mathrm{H}, \mathrm{CH}_{2} \mathrm{CH}-\mathrm{CHCH}_{2}\right), 1.53(\mathrm{~m}, 2 \mathrm{H}$, $\mathrm{CH}_{2} \mathrm{CH}_{2} \mathrm{CO}$ ), 1.51 (m, 2H, $\mathrm{CH}_{2} \mathrm{CHOH}$ ), 1.30 (br. s, $16 \mathrm{H}$, $\left.\left(\mathrm{CH}_{2}\right)_{8}\right), 0.89\left(\mathrm{t}, \mathrm{J}=6.48 \mathrm{H}_{\mathrm{Z}}, 3 \mathrm{H}, \mathrm{CH}_{2} \mathrm{CH}_{3}\right) .{ }^{13} \mathrm{C} \mathrm{NMR}$ (100 $\left.\mathrm{MH}_{\mathrm{Z}}, \mathrm{CDCl}_{3}, \delta, \mathrm{ppm}\right): 173.5(\mathrm{C}=\mathrm{O}), 148.1,147.0$ (ring), 71.7, $69.6(\mathrm{CH}-\mathrm{OH}), 69.2(\mathrm{CH}-\mathrm{NH}), 50.5\left(\mathrm{OCH}_{3}\right)$, 34.0 - 22.7 (alkyl chain), $14.1\left(\mathrm{CH}_{3}\right)$. MS (ESI): $\mathrm{m} / \mathrm{z}=$ $435.4[\mathrm{M}+\mathrm{Na}]^{+}$, Calculated $=434.1$.

\section{Conclusion}

A series of fatty ester substituted beta amino alcohol derivatives have been synthesized by the conventional heating method through ring opening of fatty esters. These derivatives were found to be very active in medicinal chemistry field as well as in the organic synthesis for future applications.

\section{Acknowledgements}

Authors are thankful to Chairman, Department of chemistry, Aligarh Muslim University for providing the necessary research facilities. Authors H. V. and A. A. are thankful to Department of Science and Technology (DST) and Council of Scientific and Industrial Research (CSIR), New Delhi respectively for the award of junior research fellowship (JRF) and also grateful to the Sophistical Analytical Instrumentation Facility (SAIF), Punjab University, for providing the spectral data. In part, the research is also supported by UGC-SAP (DRS-I).

\section{REFERENCES}

[1] S. Singh and R. Kamboj, "Synthesis of $\beta$-Amino Alcohols from Methyl Epoxy Stearate," Industrial \& Engineering Chemistry Research, Vol. 49, No. 7, 2010, pp. 3106-3111. doi:10.1021/ie901969x

[2] W. Tan, B. X. Zhao, L. Sha, P. F. Jiao and M. S. Wan, "Microwave-Assisted Ring Opening of Epoxides in Solvent-Free Conditions," Synthetic Communication, Vol. 36, No. 10, 2006, pp. 1353-1359. doi: $10.1080 / 00397910500522025$

[3] U. Das, B. Crousse, V. Kesavan, D. B. Delpon and J. P. Begue, "Facile Ring Opening of Oxiranes with Aromatic Amines in Fluoro Alcohols," Journal of Organic Chemistry, Vol. 65, No. 20, 2000, pp. 6749-6751. doi:10.1021/jo000031c

[4] F. Chemla and F. Ferreira, "Alkynyl-Oxiranes and Aziridines: Synthesis and Ring Opening Reactions with Carbon Nucleophiles," Current Organic Chemistry, Vol. 6, No. 6, 2002, pp. 539-570. doi:10.2174/1385272024604916 
[5] J. M. Chong and K. B. Sharpless, "Nucleophilic Opening of 2,3-Epoxy Acids and Amides Mediated by Titanium Isopropoxide. Highly Enhanced C-3 Selectivity," Journal of Organic Chemistry, Vol. 50, No. 9, 1985, pp. 15601563. doi: $10.1021 /$ jo00209a048

[6] M. Chini, P. Crotti, E. Giovani, F. Macchia and M. Pineschi, "Stereo and Regioselective Metal Salt-Promoted Ring Opening of 1,2-Epoxides with Thiols in Acetonitrile," Synlett, Vol. 4, 1992, pp. 303-305. doi:10.1055/s-1992-21348

[7] A. Robin, F. Brown, N. B. Rosa, B. Wu, E. Beitz, J. F. J. Kun and S. L. Flitsch, "Microwave-Assiated Ring Opening of Epoxides: A General Route to the Synthesis of 1-Aminopropan-2-ols with Anti Malaria Parasite Activities," Journal of Medicinal Chemistry, Vol. 50, No. 17, 2007, pp. 4243-4249.

[8] A. Pujala, S. Rana and A. K. Chakraboti, "Zinc Tetrafluoroborate Hydrate as a Mild Catalyst for Epoxide Ring Opening with Amines: Scope and Limitations of Metal Tetrafluoroborates and Application in the Synthesis of Antihypertensive Drugs $(R S) /(R) /(S)$-Metoprolols," Journal of Organic Chemistry, Vol. 76, No. 21, 2011, pp. 8768-8780. doi:10.1021/jo201473f

[9] G. Casiraghi, F. Zanardi, G. Rassu and P. Spanu, "Stereoselective Approaches to Bioactive Carbohydrates and Alkaloids-With a Focus on Recent Synthesis Drawing from the Chiral Pool," Chemical Review, Vol. 95, No. 6, 1995, pp. 1677-1716. doi:10.1021/cr00038a001

[10] F. R. Earle, "Epoxy Oils from Plant Seed," Journal of
American Oil Chemist's Society, Vol. 47, No. 11, 1970, pp. 510-513. doi:10.1007/BF02632975

[11] A. Biswas, K. B. Sharma, M. K. Doll, Z. S. Erhan, L. J. Willett and J. Agric, "Synthesis of an Amine-Oleate Derivative Using an Ionic Liquid Catalyst," Journal of Agricultural and Food Chemistry, Vol. 57, No. 18, 2009, pp. 8136-8141. doi:10.1021/jf901401s

[12] R. Kamboj, A. Bhadani and S. Singh, "Synthesis of $\beta$-Amino Alcohols from Terminal Epoxy Fatty Acid Methyl Ester," Industrial \& Engineering Chemistry Research, Vol. 50, No. 13, 2011, pp. 8379-8388. doi:10.1021/ie2005077

[13] N. Azizi and M. R. Saidi, "Highly Chemoselective Addition of Amines to Epoxides in Water," Organic Letters, Vol. 7, No. 17, 2005, pp. 3649-3651. doi:10.1021/o1051220q

[14] F. D. Gunstone, "Fatty Acids, Part-II. The Nature of the Oxygenated Acid Present in Vernonia anthelmintica (Wild.) Seed Oil," Journal of Chemical Society, No. 13, 1954, pp. 1611-1616. doi:10.1039/jr9540001611

[15] A. M. Davletbakova, "Catalytic Cyclopropanation of Ricinolic Acid Derivatives with Diazomethane," Russian Journal of Organic Chemistry, Vol. 37, No. 5, 2001, pp. 608-611.

[16] F. D. Gunstone and F. R. Jacobsberg, "The Preparation and Properties of the Complete Series of Methyl Epoxyoctadecanoates," Chemistry \& Physics of Lipid, Vol. 9, No. 1, 1972, pp. 26-34. 